TRANSACTIONS OF THE

AMERICAN MATHEMATICAL SOCIETY

Volume 354, Number 12, Pages 5039-5047

S 0002-9947(02)03107-0

Article electronically published on August 1, 2002

\title{
UNIFORM AND LIPSCHITZ HOMOTOPY CLASSES OF MAPS
}

\author{
SOL SCHWARTZMAN
}

\begin{abstract}
If $X$ is a compact connected polyhedron, we associate with each uniform homotopy class of uniformly continuous mappings from the real line $R$ into $X$ an element of $H_{1}\left(X, U / U_{0}\right)$, where $U$ is the space of uniformly continuous functions from $R$ to $R$ and $U_{0}$ is the subspace of bounded uniformly continuous functions. This map from uniform homotopy classes of functions to $H_{1}\left(X, U / U_{0}\right)$ is surjective. If $X$ is the $n$-dimensional torus, it is bijective, while if $X$ is a compact orientable surface of genus $>1$, it is not injective.

In higher dimensions we have to consider smooth Lipschitz homotopy classes of smooth Lipschitz maps from suitable Riemannian manifolds $P$ to compact smooth manifolds $X$. With each such Lipschitz homotopy class we associate an element of $H_{n}\left(X, B^{+} / B_{0}^{+}\right)$, where $n$ is the dimension of $P, B$ is the space of bounded continuous functions from the positive real axis to $R$, and $B_{0}^{+}$is the set of all $f \in B^{+}$such that $\lim _{t \rightarrow \infty} f(t)=0$.
\end{abstract}

\section{INTRODUCTION}

In what follows $X$ will denote a compact connected polyhedron, $R$ will be the real line equipped with its usual metric and $I$ will denote the closed unit interval. We will put the metric $\rho$ on $R \times I$ by defining $\rho\left(\left(t_{1}, s_{1}\right),\left(t_{2}, s_{2}\right)\right)$ to equal $\left|t_{1}-t_{2}\right|+\mid s_{1}-s_{2}$. Because there is a unique uniformity on $X$, the statement that a function from $R$ to $X$ or a function from $R \times I$ to $X$ is uniformly continuous has an unambiguous meaning.

Definition. If $f_{1}$ and $f_{2}$ are two uniformly continuous functions from $R$ to $X$, we will say that $f_{1}$ and $f_{2}$ are uniformly homotopic provided there exists a uniformly continuous map $H$ from $R \times I$ to $X$ such that

$$
H(t, 0)=f_{1}(t) \quad \text { and } \quad H(t, 1)=f_{2}(t) .
$$

It is clear that the relation of being uniformly homotopic is an equivalence relation on the collection of uniformly continuous functions from $R$ to $X$.

Now we will denote the additive group of uniformly continuous functions from $R$ to $R$ by $U$ and we will denote the subgroup of $U$ consisting of bounded uniformly continuous functions by $U_{0}$.

We are going to associate with each uniform homotopy class of functions from $R$ to $X$ an element of $H_{1}\left(X, U / U_{0}\right)$ and we will prove that this map from uniform homotopy classes of functions to $H_{1}\left(X, U / U_{0}\right)$ is surjective. If $X$ is the $n$-dimensional torus, we will see that this map is bijective. On the other hand, if $X$ is a compact orientable surface of genus greater than one, the map is not injective.

Received by the editors April 1, 2000 and, in revised form, May 22, 2002.

2000 Mathematics Subject Classification. Primary 54E15, 55N10. 
For any uniformly continuous function from $R$ to $X$ we will refer to the element of $H_{1}\left(X, U / U_{0}\right)$ associated with $\tilde{f}$ as the uniform homology class of $f$.

We can identify $H_{1}\left(X, U / U_{0}\right)$ with $\operatorname{Hom}\left(H^{1}(X, Z), U / U_{0}\right)$.

Recalling that $H^{1}(X, Z)$ is a finitely generated free abelian group, let $e^{1}, \cdots, e^{k}$ be an integral basis for $H^{1}(X, Z)$ and for $1 \leq i \leq k$, let $e_{i}$ be the element of $\operatorname{Hom}\left(H^{1}(X, Z), U / U_{0}\right)$ such that $e_{i}\left(e^{j}\right)=\delta_{i}^{j} \bar{\alpha}$, where $\bar{\alpha}$ is the equivalence class in $U / U_{0}$ associated with the identity map $\alpha$ of $R$ onto itself. Now suppose that $f$ is a Bohr almost periodic function from $R$ to $X$. We will show that there exist constants $\lambda_{1}, \cdots, \lambda_{k}$ such that the uniform homology class associated with $f$ equals

$$
\sum \lambda_{i} e_{i}
$$

Next we want to develop a connection with homology analogous to that above for homotopy classes of maps from a higher-dimensional space $P$ into a compact space $X$. In order to make progress, it turns out that we must assume that $P$ is a certain special kind of oriented Riemannian manifold and that $X$ is a compact smooth manifold. We will only consider smooth maps (and smooth homotopy classes of smooth maps) that satisfy a Lipschitz condition. (Note that if $\rho_{1}$ and $\rho_{2}$ are the distance functions on $X$ associated with two different Riemannian metrics, there exist positive constants $k$ and $k^{\prime}$ such that for all

$$
(x, y) \in X \times X, \quad k \rho_{1}(x, y) \leq \rho_{2}(x, y) \leq k^{\prime} \rho_{1}(x, y) .
$$

Consequently, once we have specified a Riemannian metric on $P$, the statement that a map $f$ from $P$ to $X$ satisfies a Lipschitz condition has an unambiguous meaning. If we put the usual Riemannian metric on the unit interval $I$ and then the product Riemannian metric on $P \times I$, the same is true for maps from $P \times I$ to $X$.

We will show that the relation of existence of a smooth Lipschitz homotopy between smooth Lipschitz maps of $P$ to $X$ is an equivalence relation. We will then associate with each smooth Lipschitz homotopy class of maps from $P$ to $X$ an element of $H_{n}\left(X, B^{+} / B_{0}^{+}\right)$.

Here $n$ is the dimension of $P, B^{+}$is the vector space of continuous bounded real valued functions on the positive real axis, and $B_{0}^{+}$is the subspace of functions in $B^{+}$that approach zero as we go to infinity.

We note that while it is conceivable that every smooth manifold possesses a smooth Riemannian metric satisfying the special condition that we are going to impose on $P$, it does not seem to be known if this is the case or not.

\section{The One-Dimensional Case}

In what follows $X$ will be a compact connected polyhedron. Let $C(X)$ be the multiplicative group of continuous complex-valued functions on $X$ of absolute value one and let $R(X)$ be the subgroup of $C(X)$ consisting of all functions that can be written in the form $\exp (2 \pi i \gamma(x))$ where $\gamma$ is a continuous real-valued function on $X$.

It is well known that $C(X) / R(X)$ can be identified with $H^{1}(X, Z)$.

Suppose that $f$ is a uniformly continuous function from the real line $R$ into $X$. We are going to associate with $f$ an element of $\operatorname{Hom}\left(C(X) / R(X), U / U_{0}\right)$ and we will show that this element depends only on the uniform homotopy class $f$ to which $f$ belongs. 
Let $\phi$ be any element of $C(X)$. Then there exists an $h \in U$ such that

$$
\phi(f(t))=\exp (2 \pi i h(t)) .
$$

Since $h$ is determined up to an additive constant, $\phi$ determines an element of $U / U_{0}$. If $\psi(x)=\exp (2 \pi i a(x)) \phi(x)$, then $\psi(f(t))=\exp 2 \pi i(a(f(t))+h(t))$; so $\psi$ determines the same element of $U / U_{0}$ as $\phi$. Thus, given $f$ we get a map of $C(X) / R(X)$ into $U / U_{0}$ which is easily seen to be a homomorphism.

Suppose now that $H(t, s)$ is a uniformly continuous function from $R \times I$ into $X$ such that $H(t, 0)=f(t)$ and $H(t, 1)=g(t)$. For any $\phi \in C(X), \phi(H(t, s))$ is uniformly continuous. Because $R \times I$ is contractible, there exists a continuous function $h(t, s)$ such that $\phi(H(t, s))=\exp 2 \pi i h(t, s)$. Since $\phi(H(t, s))$ is uniformly continuous, so is $h(t, s)$. It follows that $h(t, 1)-h(t, 0)$ is a bounded uniformly continuous function. Therefore $g$ determines the same element of Hom $\left(C(X) / R(X), U / U_{0}\right)$ as $f$. Since $C(X) / R(X)$ can be identified with $H^{1}(X, Z)$ and $H_{1}(X, Z)$ is finitely generated, we can identify Hom $\left(C(X) / R(X), U / U_{0}\right)$ with $H_{1}\left(X, U / U_{0}\right)$. Thus, as promised, we have associated with each uniform homotopy class of uniformly continuous functions from $R$ to $X$ an element of $H_{1}\left(X, U / U_{0}\right)$.

Since $C(X) / R(X)$ is a finitely generated free abelian group, we can get a finite set $\phi_{1}, \cdots, \phi_{k}$ of elements of $C(X)$ such that the corresponding equivalence classes $\bar{\phi}_{1}, \cdots, \bar{\phi}_{k}$ form an integral basis for $C(X) / R(X)$. Choosing a base point $p \in X$, we can select functions $x_{1}, \cdots, x_{k}$ from $I$ to $X$ such that

1. for each $i, x_{i}(0)=x_{i}(1)=p$;

2. if for each pair $(i, j), \phi_{i}\left(x_{j}(t)\right)=\exp \left(2 \pi i h_{i, j}(t)\right)$, then

$$
h_{i, j}(1)-h_{i, j}(0)=\delta_{i, j} .
$$

In other words, the closed curves on $X$ determined by the $x_{i}(t)$ yield an integral basis for $\operatorname{Hom}(C(X) / R(X), R)=H_{1}(X, R)$ dual to $\bar{\phi}_{1}, \cdots, \bar{\phi}_{k}$.

Now suppose $\lambda \in \operatorname{Hom}\left(C(X) / R(X), U / U_{0}\right)$.

We want to construct a uniformly continuous function $f$ from $R$ to $X$ such that the element of Hom $\left(C(X) / R(X), U / U_{0}\right)$ determined by $f$ is $\lambda$. We need only make certain that this element applied to each $\bar{\phi}_{i}$ yields $\lambda\left(\bar{\phi}_{i}\right)$.

Choose $u_{1}, \cdots, u_{k}$ in $U$ such that $\lambda\left(\bar{\phi}_{i}\right)=\bar{u}_{i}$, where $\bar{u}_{i}$ is the element of $U / U_{0}$ determined by $u_{i}$. For any real number $\alpha$, let $[\alpha]$ denote the largest integer less than or equal to $\alpha$ and let $N_{i, n}=\left[u_{i}(n)\right]-\left[u_{i}(n-1)\right]$.

Because the $u_{i}$ are finite in number and uniformly continuous, the set of all integers of the form $N_{i, n}$ is bounded.

For each integer $n$, let $A_{n}=\sum\left|N_{i, n}\right|$ and divide the interval $[n-1, n]$ into $A_{n}$ equal subintervals. If $N_{1, n}$ is greater than or equal to zero, define $f$ on the first $\left|N_{1, n}\right|$ subintervals of $[n-1, n]$ so that on each of these subintervals it is a translate of $x_{1}\left(A_{n} t\right)$, where $t$ ranges over the interval $\left[0,1 / A_{n}\right]$. If $N_{1, n}$ is less than zero, define $f$ on each of these subintervals to be a translate of $x_{1}\left(A_{n}(1-t)\right)$. Do the same thing on the next $\left|N_{2, n}\right|$ subintervals using $x_{2}(t)$ and so on. In this way we get a function from $[n-1, n]$ to $X$ for each integer $n$, and thus we get a function $f$ sending all of $R$ into $X$. Because $\left\{A_{n}\right\}$ is a bounded sequence, $f$ will be uniformly continuous on $R$.

We can see that $\left.\phi_{i}(f(t))\right)=\exp 2 \pi i h_{i}(t)$, where for $n \geq 1, h_{i}(n)=\sum_{1}^{n}\left(\left[u_{i}(k)\right]-\right.$ $\left.\left[u_{i}(k-1)\right]\right)=\left[u_{i}(n)\right]-\left[u_{i}(0)\right]$.

Thus for each $i, u_{i}(n)-h_{i}(n)$ is a bounded function of the integer $n$ for $n \geq 1$. It easily follows that this is a bounded function of $n$ for all integers $n$. 
Because $h_{i}(t)$ and $u_{i}(t)$ are all uniformly continuous, we can find an integer $r$ so that if $\left|t_{1}-t_{2}\right|<1 / r$, then $\left|h_{i}\left(t_{1}\right)-h_{i}\left(t_{2}\right)\right|$ and $\left|u_{i}\left(t_{1}\right)-u_{i}\left(t_{2}\right)\right|$ are less than 1 . For any $t$,

$$
\left|h_{i}(t)-u_{i}(t) \leq\right| h_{i}(t)-h_{i}([t])|+| h_{i}([t])-u_{i}([t])|+| u_{i}([t])-u_{i}(t) \mid .
$$

It follows that $\left|h_{i}(t)-u_{i}(t)\right|$ is less than $2 r+\left|h_{i}([t])-u_{i}([t])\right|$ so that $\left|h_{i}(t)-u_{i}(t)\right|$ is bounded. Therefore, the element of $\operatorname{Hom}\left(C(X) / R(X), U / U_{0}\right)$ determined by $f$ is $\lambda$.

Thus our map from uniform homotopy classes of functions from $R$ into $X$ to the corresponding uniform homology classes is surjective.

We next want to show that if $X$ is the $n$-dimensional torus $T^{n}$, our map into uniform homology classes is not only surjective but injective. We will represent $T^{n}$ as the set of all $n$-tuplets $\left(z_{1}, \cdots, z_{n}\right)$ of complex numbers of absolute value one. We will denote by $\Pi$ the map of $R^{n}$ onto $T^{n}$ sending $\left(x_{1}, \cdots, x_{n}\right)$ to $\left(z_{1}, \cdots, z_{n}\right)$ where $z_{i}=\exp 2 \pi i x_{i}$.

Suppose that $f$ and $g$ are two uniformly continuous maps of $R$ into $T^{n}$. Then we can find uniformly continuous maps $\left(a_{1}(t), \cdots, a_{n}(t)\right)$ and $\left(b_{1}(t), \cdots, b_{n}(t)\right)$ of $R$ into $R^{n}$ such that $f(t)=\left(\exp 2 \pi i a_{1}(t), \cdots, \exp 2 \pi i a_{n}(t)\right)$ and $g(t)=\left(\exp 2 \pi i b_{1}(t)\right.$, $\left.\cdots, \exp 2 \pi i b_{n}(t)\right)$.

If $f$ and $g$ determine the same element of $H_{1}\left(T_{n}, U / U_{0}\right)$, for each $i$ we must have that $a_{i}(t)-b_{i}(t)$ is a bounded function. Let $H$ be the map of $R \times I$ into $T^{n}$ defined by

$$
\begin{aligned}
H(t, s)=( & \exp \left(2 \pi i\left(a_{1}(t)+s\left(b_{1}(t)-a_{1}(t)\right)\right)\right), \\
& \left.\cdots, \exp \left(2 \pi i\left(a_{n}(t)+s\left(b_{n}(t)-a_{n}(t)\right)\right)\right)\right) .
\end{aligned}
$$

Then $H(t, s)$ is uniformly continuous and $H(t, 0)=f(t)$ while $H(t, 1)=g(t)$. Therefore $f$ and $g$ belong to the same uniform homotopy class. Thus we have shown that our map into uniform homology classes is injective.

Next we return to the situation in which $X$ is an arbitrary compact connected polyhedron. Suppose that $f$ is a Bohr almost periodic function from $R$ into $X$. If $\phi \in C(X)$, then $\phi(f(t))$ is a Bohr almost periodic function of absolute value one. If we write such a function in the form $\exp 2 \pi i h(t)$, it is well known [1] that there exists a constant $\lambda$ and a Bohr almost periodic function $a(t)$ such that $h(t)=\lambda t+a(t)$. However, since $a(t)$ is Bohr almost periodic, it is bounded. It follows that if $e_{1}, \cdots, e_{k}$ are as described in the introduction (so that $e_{i}\left(e^{j}\right)=\delta_{i, j} \bar{\alpha}$ ), then there exist constants $\lambda_{1}, \cdots, \lambda_{k}$ such that the element of $\operatorname{Hom}\left(H^{1}(X, Z), U / U_{0}\right)$ determined by $f$ is $\lambda_{1} e_{1}+\cdots+\lambda_{k} e_{k}$.

Suppose that $f$ is periodic of period one, so that it determines a closed curve in $X$ and therefore an element of $H_{1}(X, R)$. If $\alpha_{1}, \cdots, \alpha_{k}$ is the integral basis of $H_{1}(X, R)$ dual to $e_{1}, \cdots, e_{k}$, then there exist integers $n_{1}, \cdots, n_{k}$ such that this element of $H_{1}(X, R)$ equals $n_{1} \alpha_{1}+\cdots+n_{k} \alpha_{k}$. It is easy to see that the element of $\operatorname{Hom}\left(C(X) / R(X), U / U_{0}\right)$ determined by $f$ is $n_{1} e_{1}+\cdots+n_{k} e_{k}$. It follows that if $f$ and $g$ are two continuous functions of period one from $R$ to $X$, then $f$ and $g$ determine the same element of $\operatorname{Hom}\left(H^{1}(X, Z), U / U_{0}\right)$ if and only if the closed curves determined by $f$ and $g$ yield the same element of $H_{1}(X, R)$.

Now suppose that $X$ is a compact Riemannian manifold of everywhere negative sectional curvature. It is well known that each free homotopy class of closed curves (other than that of a constant curve) on such a manifold contains exactly one closed geodesic. 
Suppose that $f$ and $g$ are continuous functions of period one from $R$ to $X$ yielding closed geodesics. It is shown in [7] that if these geodesics are distinct, then $f$ and $g$ cannot be uniformly homotopic. Thus two nonconjugate elements of the fundamental group of $X$ give rise to distinct uniform homotopy classes of uniformly continuous maps from $R$ to $X$. Since it is possible for two such elements to determine the same element of $H_{1}(X, R)$, we see that our map to uniform homology classes need not be injective. In particular, since every compact orientable surface of genus greater than one admits a Riemannian metric of constant negative curvature, when $X$ is one of these surfaces, the map from uniform homotopy classes of maps to uniform homology classes will not be injective.

Finally, we want to describe the connection between asymptotic cycles as considered in 6] and uniform homology classes. To begin we need to construct a canonical homomorphism from $U / U_{0}$ to $B / B_{0}$, where $B$ is the vector space of bounded continuous functions on $R$ and $B_{0}$ is the subspace of $B$ consisting of those functions $f$ for which $\lim _{|t| \rightarrow \infty} f(t)=0$.

Suppose that $u \in U$. Since there exists an $\epsilon>0$ such that $\left|t_{1}-t_{2}\right|<\epsilon$ implies that $\left|u\left(t_{1}\right)-u\left(t_{2}\right)\right|<1$, it follows that $\lim \sup _{|t| \rightarrow \infty}\left|\frac{u(t)}{t}\right|$ is finite.

If $u(t) / t$ made sense when $t=0$, we would associate with $u$ the function $u(t) / t$.

To get around the difficulty at $t=0$, we notice that if we take two bounded open intervals $O_{1}$ and $O_{2}$ containing zero on $R$, then if $v_{1}$ and $v_{2}$ agree with $u(t) / t$ outside $O_{1}$ and $O_{2}$, respectively, then $v_{1}-v_{2}$ must certainly approach zero at infinity. Thus we associate with $u$ a unique element of $B / B_{0}$. Moreover, if $u \in U_{0}$, the element of $B / B_{0}$ we get is the zero element. Thus we get our desired homomorphism from $U / U_{0}$ to $B / B_{0}$. Composing elements of $\operatorname{Hom}\left(C(X) / R(X), U / U_{0}\right)$ with this homomorphism, we get a homomorphism from $\operatorname{Hom}\left(C(X) / R(X), U / U_{0}\right)$ to $\operatorname{Hom}\left(C(X) / R(X), B / B_{0}\right)$.

Now suppose that $f$ is a uniformly continuous function from $R$ to $X$ and let $\lambda$ be the element of $\operatorname{Hom}\left(C(X) / R(X), B / B_{0}\right)$ associated with $f$. For each $\phi \in$ $C(X) / R(X)$ choose a $b \in B$ such that $\lambda(\bar{\phi})=\bar{b}$. We are going to assume that the particular $f$ that we are considering is such that for each $\phi, \lim _{t \rightarrow \infty} b(t)$ exists. Clearly this limit depends only on $\phi$ since for $b \in B_{0}$ we have $\lim _{t \rightarrow \infty} b(t)=$ 0 . Thus for an $f$ of the type we are considering we get a map of $C(X) / R(X)$ into $R$ by sending $\phi$ into $\lim _{t \rightarrow \infty} b(t)$. Thus we associate with $f$ an element of $\operatorname{Hom}(C(X) / R(X), R)$, which we will denote by $A(f)$.

If we have a continuous flow on $X$ and $p \in X$, then in [6] we stated what it meant for $p$ to be quasi-regular and we associated with each quasi-regular point $p$ an element $A_{p}$ of $\operatorname{Hom}(C(X) / R(X), R)$ which we called the asymptotic cycle associated with $p$. A comparison of the definitions given in [6] with our present discussion shows that $A_{p}=A(f)$, where $f(t)=t p$.

\section{The Higher-Dimensional Case}

If $M$ and $X$ are smooth Riemannian manifolds and $\rho$ and $d$, respectively, are the associated distance functions on $M$ and $X$, we will say that a smooth map from $M$ to $X$ satisfies a Lipschitz condition provided there exists a constant $K$ such that for all $\left(p_{1}, p_{2}\right) \in M \times M$, we have $d\left(f\left(p_{1}\right), f\left(p_{2}\right)\right) \leq K \rho\left(p_{1}, p_{2}\right)$.

As noted in the introduction, if $X$ is compact, then given the Riemannian metric on $M$, the question of whether $f$ satisfies a Lipschitz condition or not has the same answer regardless of the particular Riemannian metric we place on $X$. 
Suppose $P$ is a complete Riemannian manifold and $X$ is compact. Put the usual Riemannian metric on the closed unit interval $I$ and put the product Riemannian metric on $P \times I$.

We will say that two smooth Lipschitz maps $f_{1}$ and $f_{2}$ from $P$ to $X$ are Lipschitz homotopic provided there exist a smooth Lipschitz map $H$ from $P \times I$ to $X$ such that

$$
H(p, 0)=f_{1}(p) \quad \text { and } \quad H(p, 1)=f_{2}(p) .
$$

It is not immediately evident that the relation of being Lipschitz homotopic between smooth Lipschitz maps is an equivalence relation, since when we string together two Lipschitz homotopies (the first from $f_{1}$ to $f_{2}$ and the second from $f_{2}$ to $f_{3}$ ) it is not necessarily true that the resulting homotopy is smooth. However, this difficulty is overcome by the following

Lemma. If $H_{1}(p, s)$ is a smooth Lipschitz homotopy from $f_{1}$ to $f_{2}$, then there exists another such Lipschitz homotopy $H_{2}(p, s)$ from $f_{1}$ to $f_{2}$ such that for $0 \leq s \leq \frac{1}{3}$, $H_{2}(p, s)=f_{1}(p)$ and for $\frac{2}{3} \leq s \leq 1, H_{2}(p, s)=f_{2}(p)$.

Proof. There exists a smooth increasing function $\gamma$ from $I$ to $I$ such that for

$$
0 \leq r \leq \frac{1}{3}, \quad \gamma(r)=0 \quad \text { and for } \quad \frac{2}{3} \leq r \leq 1, \quad \gamma(r)=1
$$

If we let $H_{2}(p, s)=H_{1}(p, \gamma(s))$, then $H_{2}(p, s)$ gives us the desired Lipschitz homotopy.

For compact smooth manifolds $X$ and suitable pairs $(P, p)$ where $p \in P$, we are going to associate with each Lipschitz homotopy class of maps from $P$ to $X$ an element of $H_{n}\left(X, B^{+} / B_{0}^{+}\right)$, where $n$ is the dimension of $P$. Here $B^{+}$is the vector space of bounded continuous real-valued functions on the positive real axis and $B_{0}^{+}$ is the subspace consisting of all $f \in B^{+}$such that $\lim _{t \rightarrow \infty} f(t)=0$.

Of course we have to say what constitutes a suitable pair $(P, p)$. First of all, $P$ will be assumed to be a smooth complete oriented Riemannian manifold. For fixed $p \in$ $P$, we will let $B_{p}(r)$ denote the set of points $q \in P$ such that $\rho(p, q) \leq r$. If it were legitimate, the condition we would like to impose on $p$ is that $\lim _{r \rightarrow \infty} A_{p}(r) / \mathrm{Vol} B_{p}(r)$ $=0$, where $A_{p}(r)$ would be defined to be the $(n-1)$-dimensional volume of the boundary of $B_{p}(r)$. Since the boundary of $B_{p}(r)$ is not necessarily an $(n-1)$ dimensional manifold, we cannot really do this. However, in [3] it is proved that for positive real numbers lying outside a certain countable set $S, B_{p}(r)$ is what is called a standard manifold in 8. This does not mean that $B_{p}(r)$ is a manifold with boundary. However, it does imply that there exists a set $N_{p}(r) \subseteq B_{p}(r)$ of $(n-1)$-dimensional Hausdorff measure zero such that the complement of $N_{p}(r)$ is a smooth manifold with boundary. Moreover, if $\omega$ is a smooth $(n-1)$-form on $P$, then $\int_{B_{p}} d \omega$ equals the integral of $\omega$ over the complement of $N_{p}(r)$ in the boundary of $B_{p}(r)$.

We will let $A_{p}(r)$ (for $r \notin S$ ) be the $(n-1)$-dimensional volume of the complement of $N_{p}(r)$ in the boundary of $B_{p}(r)$.

Definition. We will say that $(P, p)$ is a Plante pair provided that for any sequence $\left\{r_{n}\right\}$ of positive real numbers outside $S, \lim _{r \rightarrow \infty} A_{p}\left(r_{n}\right) / \operatorname{Vol} B_{p}\left(r_{n}\right)=0$.

In this situation, we will call $P$ a Plante manifold and we will refer to $p$ as a central point of $P$. It is plausible to conjecture that every point of a Plante manifold 
is a central point, but so far as the author knows it is not known if this is true or not.

We note that a condition similar to that in our definition was imposed by Plante in [4] in connection with leaves of a foliation.

Suppose next that $f$ is a smooth map from the Riemannian manifold $M$ to the Riemannian manifold $X$ such that $d\left(f\left(p_{1}\right), f\left(p_{2}\right)\right)<K \rho\left(p_{1}, p_{2}\right)$ for all $\left(p_{1}, p_{2}\right) \in$ $M \times M$.

We are going to need the following

Lemma. If $v$ is any homogeneous s-vector at the point $q \in M$, then $\left\|f_{\star} v\right\| \leq K^{s}\|v\|$. ( We need only consider the case $\|v\|=1$.)

Proof. For $s=1$ the proof is trivial. To prove the inequality for $s>1$, we first introduce smooth coordinates $\left(x_{1}, \cdots, x_{n}\right)$ about $q$ in $M$ such that $(0, \cdots, 0)$ corresponds to $q$ and at $(0, \cdots, 0)$ the Riemannian metric $g_{i j}$ equals $\delta_{i j}$. We also introduce smooth coordinates $\left(y_{1}, \cdots, y_{m}\right)$ about $f(q)$ such that $(0, \cdots, 0)$ corresponds to $f(q)$ and the Riemannian metric $h_{i j}$ from $X$ equals $\delta_{i j}$ at $(0, \cdots, 0)$.

If $v$ is a homogeneous $s$-vector, it can be represented as the exterior product of $s$ one-vectors that are mutually perpendicular; so $v=\lambda_{1} \wedge \cdots \wedge \lambda_{s}$.

Then $f_{\star} v=f_{\star}\left(\lambda_{1}\right) \wedge \cdots \wedge f_{\star}\left(\lambda_{s}\right)$.

We can assume that the coordinates $\left(x_{1}, \cdots, x_{n}\right)$ that we chose about $q$ were such that $\lambda_{1}, \cdots, \lambda_{s}$ are the unit tangent vectors corresponding to the first $s$ coordinates $x_{1}, \cdots, x_{s}$ and that the coordinates we chose about $f(q)$ were such that unit tangent vectors corresponding to the first $s$ coordinates $y_{1}, \cdots, y_{s}$ were such that the linear space they span contains each of the vectors $f_{\star} \lambda_{i}$.

Let $e_{1}, \cdots, e_{s}$ be the unit tangent vectors at $f(q)$ in $X$ corresponding to the coordinates $y_{1}, \cdots, y_{s}$.

Let $f_{\star} \lambda_{i}=\sum a_{i}^{j} e_{j}$. Then $\left\|f_{\star} \lambda_{1} \wedge \cdots \wedge f_{\star} \lambda_{s}\right\|=\left|\operatorname{det}\left(a_{i}^{j}\right)\right|$.

But a theorem of Hadamard [2] p. 1018] asserts that for any matrix $\left(a_{i j}\right)$,

$$
\left|\operatorname{det}\left(a_{i j}\right)\right| \leq \prod_{i=1}^{s}\left(\sum_{j=1}^{s}\left|a_{i j}\right|^{2}\right)^{\frac{1}{2}} .
$$

But in our present case, $\left(\sum\left|a_{i j}\right|^{2}\right)^{\frac{1}{2}}=\left\|f_{\star} \lambda_{j}\right\| \leq K$ and so $\left\|f_{\star} v\right\| \leq K^{s}$.

This proves our lemma.

Suppose next that $(P, p)$ is a Plante pair, that $X$ is a smooth compact Riemannian manifold, and that $f$ is a smooth Lipschitz map of $P$ into $X$ with Lipschitz constant $K$. Let $\left\{r_{i}\right\}$ be a sequence of positive real numbers outside the set $S$ described previously such that $\lim r_{i}=\infty$. Let $N\left(r_{i}\right)$ be the set of $(n-1)$-dimensional Hausdorff measure zero that we previously introduced. By an abuse of notation, we are going to denote the complement of $N_{p}\left(r_{i}\right)$ in the boundary of $B_{p}\left(r_{i}\right)$ by $\partial B_{p}\left(r_{i}\right)$.

Lemma. If $\omega$ is a smooth $(n-1)$-form on $X$, then

$$
\lim _{i \rightarrow \infty} \int_{B_{p}\left(r_{i}\right)} d\left(f^{\star} \omega\right) / \operatorname{Vol} B_{p}\left(r_{i}\right)=0 .
$$

Proof. We know that

$$
\int_{B_{p}\left(r_{i}\right)} d\left(f^{\star} \omega\right)=\int_{\partial B_{p}\left(r_{i}\right)} f^{\star} \omega
$$


The oriented $(n-1)$-dimensional manifold $\partial B_{p}\left(r_{i}\right)$ has on it an $(n-1)$-form $\alpha_{i}$ whose integral over any region yields the $(n-1)$-dimensional volume of that region. On $\partial B_{p}\left(r_{i}\right)$ there exists a continuous function $h_{i}$ such that on $\partial B_{p}\left(r_{i}\right),\left(J_{i}\right)^{\star}\left(f^{\star} \omega\right)=$ $h_{i} \alpha_{i}$ where $J_{i}$ is the inclusion map of $\partial B_{p}\left(r_{i}\right)$ into $P$.

Let $V_{i}$ be the field of $n-1$ vectors on $\partial B_{p}\left(r_{i}\right)$ dual to $\alpha_{i}$. Since the space of $n-1$ vectors at a point on $\partial B_{p}\left(r_{i}\right)$ is one-dimensional, $V_{i}$ is unambiguously defined. We know that

$$
\|\left(f_{\star}\left(\left(J_{i}\right)_{\star} V_{i}\right)\left\|\leq K^{n-1}\right\|\left(J_{i}\right)_{\star} V_{i} \|\right.
$$

The interior product of $f^{\star} \omega$ with $\left(J_{i}\right)_{\star} V_{i}$ equals the interior product of $\omega$ with $f_{\star}\left((J i)_{\star} V_{i}\right)$. The absolute value of this is less than or equal to

$$
K^{n-1}\left\|\left(J_{i}\right)_{\star} V_{i}\right\| \sup _{X}\|\omega\| \text {. }
$$

Since $X$ is compact, $\sup _{X}\|\omega\|$ is finite. Moreover, $\left\|\left(J_{i}\right)_{\star} V_{i}\right\|=1$. Thus there exists a single constant $C$ such that the interior product of $h_{i} \alpha_{i}$ with $V_{i}$ is less than or equal to $C$. But this just says that $\left|h_{i}\right| \leq C$ for all $i$.

Therefore, $\left|\int_{\partial B_{p}\left(\pi_{i}\right)} f^{\star} \omega\right|$ is less than or equal to $C$ times $A_{p}\left(r_{i}\right)$, where $A_{p}\left(r_{i}\right)$ is the $(n-1)$-dimensional volume of $\partial B_{p}\left(r_{i}\right)$. Since $\lim A_{p}\left(r_{i}\right) / \operatorname{Vol} B_{p}\left(r_{i}\right)=0$, it follows that

$$
\lim _{i \rightarrow \infty} \int_{B_{p}\left(r_{i}\right)} d\left(f^{\star} \omega\right) / \operatorname{Vol} B_{p}\left(r_{i}\right)=0
$$

\section{Lemma.}

$$
\lim _{r \rightarrow \infty} \int_{B_{p}(r)} f^{\star}(d \omega) / \operatorname{Vol} B_{p}(r)=0 .
$$

Proof. We need only show that for an arbitrary sequence of real numbers $\left\{r_{i}\right\}$ such that $\lim r_{i}=\infty$, it is true that

$$
\lim _{i \rightarrow \infty} \int_{B_{p}\left(r_{i}\right)} d\left(f^{\star} \omega\right) / \operatorname{Vol} B_{p}\left(r_{i}\right)=0 .
$$

However, since $S$ is countable and both $\operatorname{Vol} B_{p}(r)$ and $\int_{B_{p}(r)} d\left(f^{\star} \omega\right)$ depend continuously on $r$, whatever sequence $\left\{r_{i}\right\}$ we start with, we can get a sequence $\left\{r_{i}^{\prime}\right\}$ of numbers outside $S$ such that the difference between $\int_{B_{p}\left(r_{i}\right)} d\left(f^{\star} \omega\right) / \operatorname{Vol} B_{p}\left(r_{i}\right)$ and $\int_{B_{p}\left(r_{i}^{\prime}\right)} d\left(f^{\star} \omega\right) / \operatorname{Vol} B_{p}\left(r_{i}^{\prime}\right)$ approaches zero as $i$ goes to infinity. Thus our lemma is proved.

Next, in the situation considered above, let $\nu$ be a closed $n$-form on $X$. By an argument similar to that given above, we can show that $\left|\int_{B_{p}(r)} f^{\star} \nu / \operatorname{Vol} B_{p}(r)\right|$ is less than or equal to $K^{n} \sup _{X}\|v\|$. Thus $\int_{B_{p}(r)} f^{\star} \nu / \operatorname{Vol} B_{p}(r)$ belongs to the space $B^{+}$of bounded continuous real-valued functions on the positive real axis. By the previous lemma, if $\nu$ is a coboundary, this element of $B^{+}$belongs to the subspace $B_{0}^{+}$consisting of functions in $B^{+}$that approach zero at infinity. Thus given a smooth Lipschitz map from a Plante pair $(P, p)$ into $X$, we get an element of $\operatorname{Hom}\left(H^{n}(X), B^{+} / B_{0}^{+}\right)$, where here $H^{n}(X)$ denotes the $n^{\text {th }}$ De Rham cohomology group of $X$.

Since this can be identified with $H_{n}\left(X, B^{+} / B_{0}^{+}\right)$, it just remains to show that this element of $\operatorname{Hom}\left(H^{n}(X), B^{+} / B_{0}^{+}\right)$depends only on the Lipschitz homotopy class of $f$ and not on $f$ itself. 
Suppose that $(P, p)$ is a Plante pair, $X$ is a smooth compact Riemannian manifold, and $H$ is a smooth Lipschitz function from $P \times I$ to $X$. If $f_{1}(p)=H(p, 0)$ and $f_{2}(p)=H(p, 1)$, we have to prove that the elements of $\operatorname{Hom}\left(H^{n}(X), B^{+} / B_{0}^{+}\right)$ determined by $f_{1}$ and $f_{2}$ are the same. (Here $n$ of course is the dimension of $P$.)

By Stoke's theorem, if $\nu$ is a closed smooth $n$-form on $X$, the difference be-

tween $\int_{B_{p}(r)} f_{1}^{\star}(\nu)$ and $\int_{B_{p}(r)} f_{2}^{\star}(\nu)$ equals the integral of $H^{\star}(\nu)$ over the Cartesian product of $\partial B_{p}(r)$ with $I$ (provided $r$ does not belong to the countable set $S$ ).

We need only show that the absolute value of this integral divided by $\operatorname{Vol}\left(B_{p}(r)\right)$ approaches zero as $r$ goes to infinity to get the result we want. However, the absolute value of this integral is less than or equal to $K^{n} \sup \|\nu\|$ times the $(n-1)$ dimensional volume of $\partial B_{p}(r)$, which we have denoted by $A_{p}(r)$. By the definition of a Plante pair,

$$
\lim _{r \rightarrow \infty} A_{p}(r) / \operatorname{Vol} B_{p}(r)=0 .
$$

Thus we have finally associated with each Lipschitz homotopy class of maps from $P$ to $X$ an element of $H\left(X, B^{+} / B_{0}^{+}\right)$.

If we take $P$ to be the real line with its usual Riemannian metric and $p$ to be $0,(P, p)$ is a Plante pair. The present treatment associates with each Lipschitz homotopy class of maps from this $P$ to $X$ an element of $H_{1}\left(X, B^{+} / B_{0}^{+}\right)$. However, each Lipschitz homotopy class of maps from this $P$ to $X$ determines a uniform homotopy class of maps from this $P$ to $X$.

In our treatment of the one-dimensional case, we associated with such a uniform homotopy class an element of $H_{1}\left(X, B / B_{0}\right)$. The coefficient homomorphism from $B / B_{0}$ to $B^{+} / B_{0}^{+}$induced by the homomorphism from $B$ to $B^{+}$that assigns to each $f \in B$ the element of $B^{+}$whose value at any $x$ is $\frac{1}{2}(f(x)+f(-x))$ induces a map of $H_{1}\left(X, B / B_{0}\right)$ to $H_{1}\left(X, B^{+} / B_{0}^{+}\right)$sending our homology class into the correct element of $H_{1}\left(X, B^{+} / B_{0}^{+}\right)$.

\section{REFERENCES}

[1] H. Bohr, Überfast Periodischen Bewegungen, In his collected mathematical works, Vol. 2, Dansk. Mat. Forening-Copenhagen, C44 (1952). MR 15:276i

[2] N. Dunford and J. Schwartz, Linear Operators Part II, Spectral theory. Selfadjoint operators in Hilbert space, Interscience Publishers, (1963). MR 32:6181

[3] R. Moussu and F. Pelletier, Sur le Théorème de Poincaré-Bendixson, Annales Institute Fourier, Vol. 24 (1974), pp. 131-148. MR 50:11266

[4] J. F. Plante, A Generalization of the Poincaré-Bendixson Theorem for Foliations of Codimension One, Topology, Vol. 12 (1973) pp. 177-181. MR 49:6253

[5] J. F. Plante, Foliations with Measure Preserving Holonomy, Annals of Math., Vol. 102 (1975), pp. 327-361. MR 52:11947

[6] S. Schwartzman, Asymptotic Cycles, Annals of Math., Vol. 66 (1957) pp. 270-284. MR 19:568i

[7] S. Schwartzman, Bohr Almost Periodic Maps into $K(\pi, 1)$ Spaces, Proceedings Amer. Math. Soc., Vol. 125 (1997), pp. 427-431. MR 97d:58163

[8] H. Whitney, Geometric Integration Theory, Princeton University Press, Princeton, NJ (1957). MR 19:309c

Department of Mathematics, University of Rhode Island, Kingston, Rhode Island 02881 